\title{
Was? Wie? Warum? Jugendliche erforschen Flussland- schaften - Förderung des Systemverständnisses als Basis für gelebte Partizipation im Flussgebietsmanagement
}

\author{
Michaela Poppe · Kerstin Böck · Andreas Zitek · Sigrid Scheikl · Andreas Loach · Susanne Muhar
}

Online publiziert: 12. Juli 2016

(c) Der/die Autor(en) 2016. Dieser Artikel ist eine Open-Access-Publikation.

\begin{abstract}
Zusammenfassung Flusslandschaftsplanung und -management im Sinne einer zukunftsverträglichen Entwicklung einer Flusslandschaft sind dem Prinzip der Nachhaltigkeit verpflichtet. Ziel ist es dabei, eine Entwicklung von Flusslandschaften $\mathrm{zu}$ initiieren, die die Anforderungen des Natur- und Gewässerschutzes mit den sozialen und wirtschaftlichen Ansprüchen in Einklang bringt und die betroffenen BürgerInnen in den Entscheidungsprozess einbezieht. Systemverständnis und ökologische Handlungskompetenz sind für Beteiligungsprozesse wesentliche Voraussetzungen.
\end{abstract}

Im Oktober 2014 startete im Rahmen des Forschungsprogrammes „Sparkling Science" des Bundesministeriums für Wissenschaft, Forschung und Wirtschaft das Projekt „Traisen. $w^{3}$ - Traisen. Was? Wie? Warum? - Identifizierung und Wahrnehmung von Funktionen in Flusslandschaften und Verstehen einzugsgebietsbezogener Prozesse am Beispiel der Traisen“. In diesem Projekt arbeitet ein WissenschaftlerInnen-Team gemeinsam mit SchülerInnen und PädagogInnen zweier Oberstufenklassen des BG/BRG St. Pölten über einen Zeitraum von zwei Jahren zu Forschungsfragen des Flusslandschaftsmanagements am Beispiel der Traisen. Neben der Integration der SchülerInnen in den

Mag. Dr. M. Poppe $(\bowtie) \cdot$ DI K. Böck •

DI S. Scheikl · DI A. Loach .

ao.Univ.-Prof. DI Dr. S. Muhar

Institut für Hydrobiologie

und Gewässermanagement,

Universität für Bodenkultur Wien,

Max-Emanuel-Straße 17, 1180 Wien,

Österreich

E-Mail: michaela.poppe@boku.ac.at

DI Dr. A. Zitek, MSc.

Abteilung für Analytische Chemie, Universität für Bodenkultur Wien, Konrad Lorenz-Straße 24/II, 3430 Tulln an der Donau, Österreich
Forschungsprozess, in Freilandarbeiten und Datenauswertungen und -interpretationen ist vor allem die Entwicklung von Systemverständnis in Bezug auf Prozesse in Flusslandschaften Ziel des Projektes.

Die SchülerInnen wurden mithilfe der Lern-Software „DynaLearn“ angeregt, sich konzeptuelles und kausales Wissen anzueignen und damit systemische Prozesse in der Umwelt zu verstehen und in qualitative Modelle umzusetzen. Als weiteres Instrument, die Komplexität von Flusslandschaften anzusprechen, wurde das Konzept der Ökosystemleistungen verwendet. In Wissenstests sowie durch die Auswertung der qualitativen SchülerInnenModelle wurden das Wissen und das Systemverständnis der Schülerinnen vor und nach den gemeinsamen Aktivitäten evaluiert.

Die Auswertungen zeigten, dass regionales Wissen über den Heimatfluss und Vorgänge im Einzugsgebiet nicht im Bewusstsein der Jugendlichen verankert sind. Aufgrund der durchgeführten Schul-Workshops konnte ein signifikanter Zuwachs des Wissens und Verständnisses der SchülerInnen festgestellt werden. Die Modelle der SchülerInnen zeigten die komplexen Fragestellungen im Flussgebietsmanagement auf. Alle Modell-Szenarien der Jugendlichen spiegelten nachhaltige Entwicklungen der Flusslandschaft wider und belegten ein maßgeblich gesteigertes Verständnis der SchülerInnen für wichtige kausale Zusammenhänge an der Traisen.

Die Ergebnisse dokumentieren weiters, dass das Konzept der Ökosystemleistungen eine geeignete Methode zur Wissensvermittlung von komplexen Zusammenhängen in Flusslandschaften darstellt. Die Erhebung der Wahrnehmung von Ökosystemleistungen durch BürgerInnen, insbesondere Jugendliche, kann zu einer umfassenden Betrachtung der Flusslandschaft in zukünftigen Planungen im Flusslandschaftsmanagement beitragen. Mittels der in diesem Rahmen identifizierten Wissenslücken und des fehlenden Umweltverständnisses kann der zukünftige Bildungsbedarf hinsichtlich Funktionsfähigkeit und Leistungen von Flusslandschaften abgeschätzt werden. Gerade bei jungen Menschen ist es wichtig, das Interesse für Umwelthemen $\mathrm{zu}$ fördern und eine mögliche Beteiligung an Entscheidungen zu forcieren. Gezielte Initiativen sind notwendig, damit junge Menschen zukünftig als BewohnerInnen und NutzerInnen der Flusslandschaften nachhaltige Planungen und Maßnahmen unterstützen, sich aktiv an der Entscheidungsfindung beteiligen und damit Verantwortung für die Gestaltung ihrer Umwelt übernehmen.

Schlüsselwörter Systemverständnis . Flusslandschaft .

Einzugsgebietsmanagement .

Ökosystemleistungen .

Wissensvermittlung · Partizipation

What? How? Why? Students explore river landscapes promoting a systems-based understanding as the cornerstone of "lived" participation in river management

Abstract River landscape planning and management to promote the positive long-term development of river landscapes must adhere to the principle of sustainability. In this context, the goal is to initiate a development process for river landscapes that successfully reconciles the requirements of nature and water conservation with social and economic aspects, and which actively involves affected citizens in the decision-making process. A systems-based understanding and the opportunity to make ecologically relevant decisions are 
essential prerequisites for participative processes.

In the context of the Austrian Federal Ministry of Science, Research and Economy research program "Sparkling Science," the project "Traisen. $\mathrm{w}^{3}$ - Traisen. What?How?Why? - Identification and Perception of Functions in River Landscapes and Understanding Catchmentrelated Processes on the Example of the Traisen River" was launched in October 2014. The project involves a team of scientists working together with teachers and students from two high-school classes from the BG/BRG St. Pölten schools over a two-year period to investigate central research questions on river landscape management using the example of the Traisen River. Beyond integrating the students in the research process, in field work, and in assessing and interpreting results, one of the project's central goals is to promote a systems-based understanding of the processes at work in river landscapes.

With the help of the learning software program "DynaLearn," students were encouraged to acquire both conceptual and problem-solving skills, which in turn allowed them to better understand systematic processes in the environment and implement those processes in qualitative models. The concept of ecosystem services was employed as a further means of approaching the complexity of river landscapes. Using knowledge-based tests and by assessing their qualitative models, the students' systems-based understanding before and after their shared activities was evaluated.

The results show that regional knowledge of their home river and key processes in its catchment area is not anchored in the students' minds. The school workshops confirmed a significant increase in the students' knowledge and grasp of the subject matter, while the models they produced illustrate the complex questions involved in river landscape management. All of the model scenarios the students depicted reflect sustainable developments in the river landscape and evince their considerably improved grasp of important causal relationships on the Traisen.

The results also confirm that the concept of ecosystem services offers a suitable method for conveying complex interrelationships in river landscapes. Raising awareness of ecosystem services among citizens, and especially among young people, can help to ensure that more comprehensive approaches are utilized in future river landscape planning and management efforts. Further, the gaps in knowledge and lack of environmental awareness identified in the course of the project can be used to determine the future need for education on the functions of and ecosystem services provided by river landscapes. When it comes to young people, it is essential that we promote their interest in environmental issues and offer them opportunities to join in decision-making processes. Focused initiatives are called for to ensure that these future inhabitants and users of river landscapes support sustainable planning and measures, actively participate in decision-making, and in so doing take on responsibility for shaping their own environment.

Keywords Systems-based understanding · River landscape . Catchment management · Ecosystem services · Knowledge acquisition . Participation

\section{Einleitung}

Systemverständnis und Handlungskompetenz in komplexen Systemzusammenhängen sind wesentliche Voraussetzungen für das Treffen von nachhaltigen Entscheidungen in unserer vernetzten Welt (Frischknecht-Tobler et al. 2008). Die 2030-Agenda für eine nachhaltige Entwicklung (UN 2015) beschreibt klare Zusammenhänge zwischen einer nachhaltigen Entwicklung, der Notwendigkeit eines umfassenden Systemverständnisses und neuen gesellschaftlichen Lösungsansätzen (Bückmann 2015).

Demgegenüber stehen die Ergebnisse der PISA-Studie aus 2006 (OECD 2009), die bei 15-jährigen Jugendlichen ein Basiswissen zu Umwelthemen dokumentierten, allerdings klar ein fehlendes Verständnis und geringes Wissen bei den jungen Menschen aufzeigten, wie Umweltprobleme zukünftig gelöst werden könnten. Auch Evaluierungsergebnisse von Wissenstests bei 15bis 17-jährigen SchülerInnen belegten, dass das Verständnis für komplexe $\mathrm{Zu}$ sammenhänge gewässerökologischer Themen wenig ausgeprägt ist (Poppe et al. 2013).

Im Oktober 2014 startete im Rahmen des Forschungsprogrammes „Sparkling Science" des Bundesministeriums für Wissenschaft und Forschung das Pro- jekt „Traisen.w $w^{3}$ - Traisen. Was? Wie? Warum? - Identifizierung und Wahrnehmung von Funktionen in Flusslandschaften und Verstehen einzugsgebietsbezogener Prozesse am Beispiel der Traisen“1. In diesem Projekt arbeitet ein WissenschaftlerInnen-Team gemeinsam mit SchülerInnen und PädagogInnen zweier Oberstufenklassen des BG/BRG St. Pölten über einen Zeitraum von zwei Jahren zu Forschungsfragen des Flusslandschaftsmanagements. Inhaltlich baut das Projekt auf Erkenntnissen des Vorgängerprojektes „FlussAu:WOW!“ auf (Poppe et al. 2013). Neben der Integration der SchülerInnen in den Forschungsprozess, in Freilandarbeiten und Datenauswertungen und -interpretationen ist vor allem der Aufbau von Systemverständnis in Bezug auf Prozesse in Flusslandschaften Ziel des Projektes. Systemverständnis wird nach Frischknecht-Tobler et al. (2008) als „Fähigkeit, komplexe Wirklichkeitsbereiche als Systeme zu beschreiben, $z u$ rekonstruieren und zu modellieren sowie Erklärungen zu geben, Prognosen zu treffen und Handlungsmöglichkeiten $z u$ entwerfen und zu beurteilen "definiert.

Als Werkzeug der Wissensvermittlung und vor allem um das Systemverständnis für Prozesse in Flusslandschaften zu vertiefen, eignet sich das Konzept des qualitativen Modellierens (Bredeweg et al. 2013; Zitek et al. 2009). Im Projekt „Traisen.w ${ }^{3 “}$ wurden die SchülerInnen angeregt, sich mithilfe der Lern-Software „DynaLearn“ ${ }^{2}$ selbständig konzeptuelles und kausales Wissen anzueignen und damit systemische Prozesse in der Umwelt zu verstehen und in qualitative Modelle umzusetzen. Bereits durchgeführte Evaluierungen dieses Ansatzes mit SchülerInnen einer berufsbildenden höheren Schule haben gezeigt, dass es durch die Arbeit mit der Lern-Software zu einem signifikanten Anstieg von kausal richtigen Beziehungen bei den Antworten zu fachlichen Wissensfragen kam (Zitek et al. 2013; Poppe et al. 2011). Durch diese Möglichkeit der Darstellung von Umweltphänomenen mittels Modellierungsinstrumenten können „Wissenschaftsneulinge“ eine Art von Problemlösungsverhalten von ExpertInnen erleben und erlernen (Jonassen 2003).

\footnotetext{
1 http://www.traisen.net/

2 http://www.dynalearn.eu.
} 
Ein weiteres Instrument, die Komplexität von Flusslandschaften anzusprechen, sie "greifbarer" und damit operationalisierbar zu machen, stellt das Konzept der Ökosystemleistungen (ÖSL) dar. Dieses behandelt die Schnittstelle zwischen Ökosystemen und menschlichem Wohlbefinden und rückt die von Ökosystemen bereitgestellten Güter und Leistungen in den Vordergrund, die zum menschlichen Wohlbefinden beitragen können. Diese können in Versorgungsleistungen (z. B. Verfügbarkeit von Wasser und Rohstoffen), Regulierungsleistungen (z. B. Hochwasserschutz), kulturelle Leistungen (z. B. Erholungsleistung) sowie unterstützende Basisleistungen (ökologische Leistungen, z. B. Erfüllung der Habitatfunktion) eingeteilt werden (MEA 2005). Vor dem Hintergrund sich ständig wandelnder Nutzungsansprüche an unsere Landschaft sind sowohl ökologische als auch kulturelle Funktionen und Leistungen von besonderem Interesse. Gerade letztere spielen für Kinder und Jugendliche, als eine der größten NutzerInnengruppen natürlicher Freiflächen (Millward and Mostyn 1989; Tapsell et al. 2001), eine große Rolle. In der Praxis, zum Beispiel im Rahmen von Kulturlandschaftsbewertungen, finden diese jedoch meist noch wenig Berücksichtigung (Schaich et al. 2010). Um kulturelle ÖSL in Managementkonzepte einfließen $\mathrm{zu}$ lassen, wird von Chan et al. (2012) vorgeschlagen, soziokulturelle Methoden in ÖSLBewertungen einzubinden. Besonders die Sichtweisen von Kindern und Jugendlichen könnten in diesem Rahmen dazu beitragen, die Nutzbarkeit von Fließgewässern z. B. für Erholungszwecke zu verbessern (Tapsell et al. 2001). Die meisten Untersuchungen bisher beschränken sich jedoch auf StakeholderGruppen mit gewissen Entscheidungskompetenzen an Fließgewässern (Böck et al. 2013) bzw. erwachsene BewohnerInnen eines betroffenen Gebiets (Plieninger et al. 2013). Bis dato gibt es nur vereinzelte am ÖSL-Konzept orientierte, wissenschaftliche Untersuchungen, die sich mit der Wahrnehmung von Jugendlichen in Flusslandschaften befassen (z. B. Chuchmáková 2009; Eder und Arnberger 2010).

Die Erhebung der Wahrnehmung von ÖSL durch BürgerInnen, insbesondere Jugendliche, kann zu einer umfassenden Betrachtung der Flusslandschaft in zukünftigen Planungen im Flusslandschaftsmanagement bei- tragen. Mittels der in diesem Rahmen identifizierten Wissenslücken kann der zukünftige Bildungsbedarf hinsichtlich Funktionsfähigkeit und Leistungen von Flusslandschaften abgeschätzt werden. Seit der Entstehung des ÖSLKonzepts gibt es Bemühungen, dieses in Bildungsaktivitäten einzubauen ${ }^{3}$. So wird beispielsweise auf UN-Ebene das ÖSL-Konzept in bestehende Bildungsprogramme (z. B. SWEDESD ${ }^{4}$ ) eingebunden. Es soll in diesem Zusammenhang dazu beitragen, das Verständnis von Kindern und Jugendlichen für den Nutzen sichtbar zu machen, den Menschen aus intakten Ökosystemen ziehen, und damit die Bedeutung einer nachhaltigen Entwicklung zu unterstreichen. Auch im vorliegenden Projekt „Traisen.w. ${ }^{3{ }^{34}}$ wird das Konzept der ÖSL als Kommunikations- und Bildungswerkzeug in der Zusammenarbeit mit SchülerInnen eingesetzt.

Flusslandschaftsplanung und -management im Sinne einer zukunftsverträglichen Entwicklung einer Flusslandschaft sind dem Prinzip der Nachhaltigkeit verpflichtet. Ziel ist es dabei, eine Entwicklung von Flusslandschaften zu initiieren, die Anforderungen des $\mathrm{Na}$ tur- und Gewässerschutzes mit den sozialen und wirtschaftlichen Ansprüchen der betroffenen Menschen in Einklang bringt. Umweltwissen und Systemverständnis sind zentrale Grundlagen für eine nachhaltige Entwicklung (UNESCO 2011; Bilharz und Gräsel 2006). Auch im Flussgebietsmanagement, wo komplexe Zusammenhänge $u$. a. zwischen den Bereichen Wasserwirtschaft, Ökologie, Landschaftsplanung, Siedlungsentwicklung, Land- und Forstwirtschaft, Wirtschaft, Naturschutz und Tourismus bestehen, sind nachhaltige Entscheidungen und Lösungen unabdingbar. Um gemeinsame Entscheidungen $\mathrm{zu}$ treffen, Entscheidungsprozesse auf eine breite Basis $\mathrm{zu}$ stellen und die Akzeptanz der Entscheidungen zu erhöhen, ist die Information und Partizipation sowohl von EntscheidungsträgerInnen, Akteursgruppen als auch von der breiten Öffentlichkeit notwendig. Die Information und Beteiligung der Bevölkerung an Entscheidungsprozessen ist durch mehrere EU-Richtlinien (u. a. Wasserrahmenrichtlinie: Richt-

\section{3 http://www.es-partnership.org/esp/} $82584 / 5 / 0 / 50$

${ }^{4}$ Swedish International Centre of Education for Sustainable Development. linie 2000/60/EG, Hochwasserrichtlinie: Richtlinie 2007/60/EG, Richtlinie über die Beteiligung der Öffentlichkeit: Richtlinie 2003/35/EG) gefordert und festgelegt. Daher ist es wichtig, dass sich Jugendliche der Notwendigkeit einer nachhaltigen Entwicklung von Flusslandschaften bewusst sind und als zukünftige BewohnerInnen und NutzerInnen dieser Landschaften nachhaltige Planungen, Maßnahmen und Entscheidungen unterstützen. Umweltbildung und gelebte Partizipation bedingen einander und unterstützen sich gegenseitig.

Der vorliegende Beitrag befasst sich mit der Förderung von Systemverständnis und Umweltwissen zu Prozessen in Flusslandschaften im Rahmen des Projektes „Traisen.w ${ }^{3{ }^{4}}$. Die Ergebnisse der Wissensevaluierung von Ursache-Wirkungs-Zusammenhängen in Flusslandschaften, des Konzepts der ÖSL und zum regionalen Wissen zur Traisen liegen bereits vor und sind hier zentrales Thema. Ziel ist es, folgende Fragen zu beantworten: 1) Können SchülerInnen durch die Arbeit im Forschungsprojekt mit einer Lern-Software und Arbeiten im Feld verstärkt systemare Zusammenhänge in Umweltfragen herstellen? und 2) Inwiefern ist das ÖSL-Konzept zur Anwendung als Kommunikationsinstrument und zur Verbesserung des Systemverständnisses bei Jugendlichen geeignet?

\section{Vorgangsweise und Methodik}

\subsection{Durchführung der Schüleraktivitäten}

Mit dem Kick-off-Workshop im Oktober 2014 startete für die 5 . und 7. Klasse des naturwissenschaftlichen Zweiges $(5 \mathrm{~N}$ und $7 \mathrm{~N}$ ) des Gymnasiums St. Pölten die Forschungsmitarbeit im Sparkling-

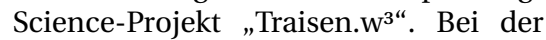
Zusammenarbeit mit den SchülerInnen wurde neben der Wissensvermittlung besonderes Augenmerk auf die Förderung des Verständnisses der SchülerInnen für komplexe Zusammenhänge in Flusslandschaften gelegt. In multimodalen Workshops wurden die SchülerInnnen in die Forschungsthemen eingeführt (Tab. 1).

\subsubsection{Modellierungs-Workshops}

Die SchülerInnen der 7. Klasse waren bereits im Vorgängerprojekt „FlussAu: WOW!“ (Poppe et al. 2013) tätig und arbeiteten dort mit der Lern-Software 
Tab. 1 Überblicküber zeitliche Abfolge und Inhalte der Schul-Aktivitäten inkl. der Wissensevaluierungen im Projekt ,Traisen.w w $^{34}$ im ersten Schuljahr 2014/2015

\begin{tabular}{|c|c|c|c|c|}
\hline Aktivitäten & Klasse & Wissensevaluierung & Datum & Thema \\
\hline Kick-off-Workshop & $5 \mathrm{~N} / 7 \mathrm{~N}$ & Prä-Test (T1) & 07.11 .2014 & Vorstellung, Projektvorschau \\
\hline 1. Workshop zu ÖSL & $5 \mathrm{~N}$ & & 27.01 .2015 & Flusslandschaften, ÖSL, Flusscharakteristik Traisen \\
\hline 1. Modellierungs-Workshop & $7 N$ & & 29.01.2015 & $\begin{array}{l}\text { Flusscharakteristik Traisen, „DynaLearn“-Modellieren: } \\
\text { Ursache-Wirkungs-Zusammenhänge im Einzugsgebiet } \\
\text { Traisen }\end{array}$ \\
\hline 2. Modellierungs-Workshop & $7 \mathrm{~N}$ & Mid-Test (T2) & 10.02 .2015 & $\begin{array}{l}\text { Belastungen/Renaturierungen im Traisen Einzugsgebiet, } \\
\text { „DynaLearn“-Modellieren: Entwicklung von Szenarien im } \\
\text { Einzugsgebiet Traisen }\end{array}$ \\
\hline 2. Workshop zu ÖSL & $5 \mathrm{~N}$ & Mid-Test (T2) & 13.02.2015 & Wissenschaftliche Methode, Google Earth-Kartierung ÖSL \\
\hline Gemeinsamer Aktionstag & $5 \mathrm{~N} / 7 \mathrm{~N}$ & Post-Test (T3) & 25.06 .2015 & $\begin{array}{l}\text { Feldarbeiten in Kleingruppen (Bereich St. Pölten und Trai- } \\
\text { senunterlauf nahe Traismauer), Besichtigung des LIFE+ } \\
\text { Projektes Traisen }\end{array}$ \\
\hline
\end{tabular}

„DynaLearn“. Darauf aufbauend wurden im ersten Modellierungs-Workshop Expertenmodelle als „Rahmenmodelle" vorgestellt, die zur Beschreibung typischer Prozesse in Flusslandschaften entwickelt wurden. Basierend auf diesen Modellen konnten regionale Modelle, die für das Traisen-Einzugsgebiet gültig sind, entwickelt werden. Die SchülerInnen erstellten in Kleingruppen von 2 bis 3 Personen qualitative Modelle zum Zustand der Traisen. Sie setzten Ursache-Wirkungs-Zusammenhänge in Flusslandschaften am Beispiel ihres Heimatflusses in kausale Modelle um. Im zweiten Workshop verfassten die SchülerInnen eine textliche Version eines potenziellen Entwicklungsszenarios für das Einzugsgebiet der Traisen. Darauf aufbauend wurde ein entsprechendes Modell konstruiert. Das Modellieren diente vor allem der Unterstützung bei der Ausformulierung der Szenarien. Zur Orientierung wurden den SchülerInnen drei Hauptfragen zur Entwicklung der Szenarien gestellt: (1) Was ist das Hauptproblem an der Traisen, das ihr modellieren wollt? (2) Wie soll sich der Zustand verändern? Und (3) Wo müsste man vermutlich ansetzen?

\subsubsection{Workshops zu ÖSL}

Im Zuge der Workshops mit dem thematischen Schwerpunkt „Ökosystemleistungen“ sollten SchülerInnen der 5. Klasse einen Einblick in die verschiedenen Funktionen und Nutzungsformen von Fließgewässern, insbesondere der Traisen erhalten. Der Fokus wurde dabei auf „unterstützende Basisleistungen“ (z. B. Fluss-Lebensraum) und kulturelle Leistungen (u. a. Bewegung, Naturerlebnis) gelegt. In Kleingruppen erarbeiteten die SchülerInnen verschie- dene Themengebiete im Kontext von ÖSL in Flusslandschaften und präsentierten diese dann ihren MitschülerInnen. Im folgenden Workshop erstellten die SchülerInnen digitale Karten in Google Earth, in denen sie ÖSL des Einzugsgebiets der Traisen identifizierten und in der Karte abbildeten.

\subsubsection{Feldarbeiten}

Beim Aktionstag im Juni 2015 kartierten die SchülerInnen beider Klassen gemeinsam an zwei Abschnitten der Traisen ausgewählte Indikatoren für ökologische (z. B. Sedimentbänke, Uferstrukturen) und kulturelle Ökosystemleistungen (u. a. Radwege, Sitzgelegenheiten am Ufer, Infotafeln). Danach konnten sie im Rahmen einer Führung das Projektgebiet des LIFE+ Projekts Traisen und die dort neu entstandene Flusslandschaft besichtigen.

\subsection{Durchführung und Auswertungen der Wissensevaluierungen}

$\mathrm{Zu}$ Beginn des Projekts erfolgte der erste Wissenstest (Prä-Test; T1) während der Kick-off-Veranstaltung in den beiden Klassen $5 \mathrm{~N}$ und $7 \mathrm{~N}$. In der $5 \mathrm{~N}$ wurde der Mid-Test (T2) nach den beiden Workshops über ÖSL durchgeführt, in der $7 \mathrm{~N}$ nach zwei ModellierungsWorkshops (siehe Tab. 1). Insgesamt absolvierten 47 SchülerInnen alle drei Tests. Die SchülerInnen beantworteten jeweils die gleichen Fragen zu den Themenblöcken „regionales Wissen“, „Systemverständnis“ und „ÖSL“. Die Antworten folgender Fragen (Block A regionales Wissen, Block B - Systemverständnis) wurden für diesen Beitrag mit einer Punktebewertung analysiert:
- A1) „Wodurch ist der Zustand der Traisen deiner Meinung nach beeinträchtigt?" und

- A2) „Kennst du Renaturierungsprojekte im Traisen-Einzugsgebiet? Wenn ja, welche?"

- B1) „Warum wurden/werden Flüsse reguliert? Was verändert sich dadurch (für Menschen, Tiere und Pflanzen)?" und

- B2) „Warum werden Flüsse renaturiert? Was verändert sich dadurch (für Menschen, Tiere und Pflanzen)?“ Bei den Fragen A1), B1) und B2) konnten maximal 4 Punkte erreicht werden, bei A2) 2 Punkte.

Zusätzlich wurden die Antworten auf die Frage A3) „In welchem ökologischen Zustand befindet sich die Traisen im Bereich St. Pölten deiner Meinung nach?" nach den Zustandsklassen analysiert sowie die einzelnen Antworten auf die Frage A1) Beeinträchtigungstypen zugeordnet und in einem Balkendiagramm dargestellt.

Die Antworten auf die Frage C1) „Welchen Nutzen haben Flusslandschaften für den Menschen?" wurden den vier ÖSL-Hauptklassen (unterstützende-ökologische Basisleistungen, kulturelle, regulierende und versorgende ÖSL) entsprechend der Einteilung nach MEA (2005) kategorisiert und ausgewertet.

\subsection{Auswertung qualitativer Modelle und Szenarien}

Die SchülerInnen-Modelle wurden in Hinblick auf das Erlernen einer kausalen, qualitativen Ausdrucksweise, die das systemare Verständnis fördern soll, evaluiert. Der in „DynaLearn“ umgesetzte qualitative kausale Ansatz, der vom generellen sprachlichen Verständnis ausgeht, wurde dabei durch Ele- 


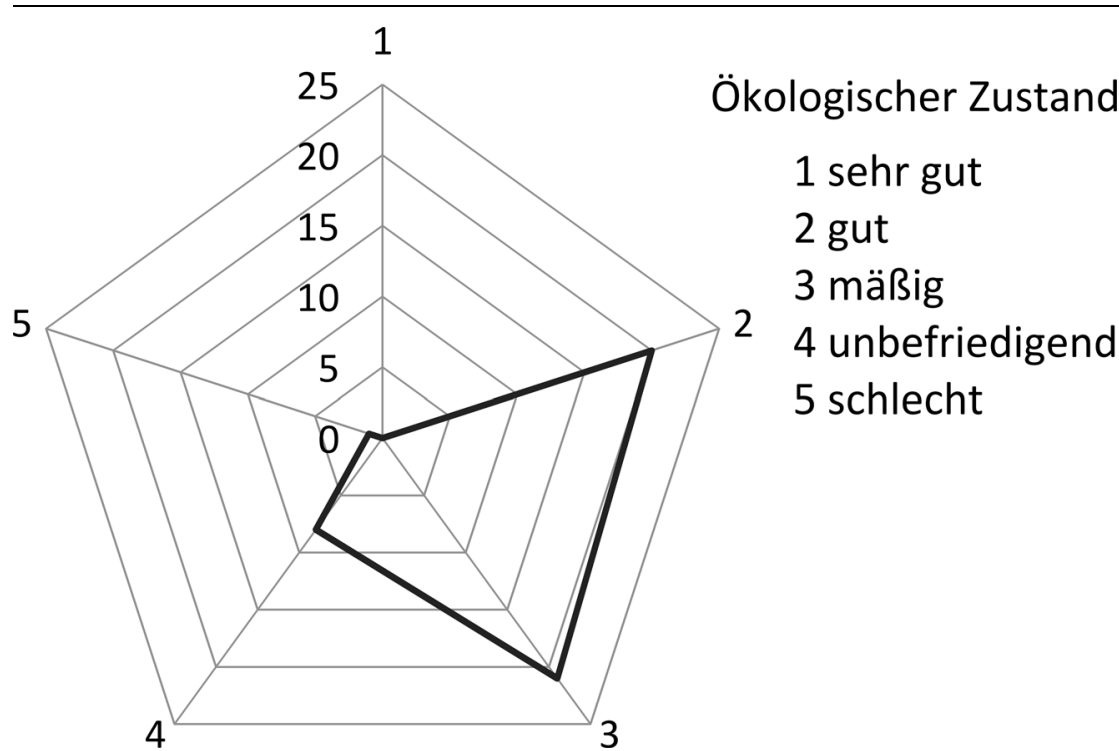

Abb. 1 Einschätzung desökologischenZustands derTraisenimBereichvonSt.Pölten durch die SchülerInnen im Prä-Test, $N=51$

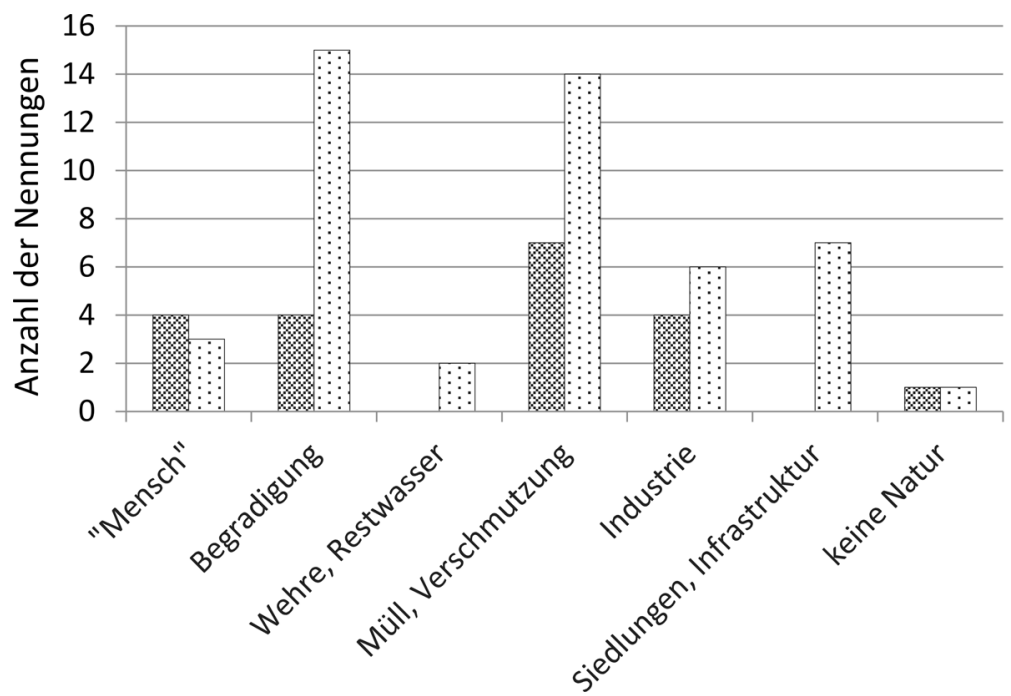

霖 $5 \mathrm{~N}$

Abb. 2 Antworten der SchülerInnen beim Prä-Test(T1) auf die Testfrage A1),,Wodurch ist der Zustand der Traisen deiner Meinung nach beeinträchtigt?" differenziert nach Belastungstypen

mente der qualitativen Systemdynamik erweitert (Zitek et al. 2013). Die erstellten SchülerInnen-Modelle wurden im Detail auf deren wesentliche Elemente und Zusammenhänge hin analysiert und mit den ExpertInnen-Modellen verglichen. Evaluierungskriterien waren (1) die Verwendung wesentlicher Hauptelemente und deren hierarchische Beziehung und (2) Richtigkeit der Terminologie, der kausalen Beziehungen und der Darstellung von Prozessen in Flusslandschaften.

Die am Ende von den SchülerInnen erstellten Szenarien sollten letztend- bar waren, da eines von der Software nicht mehr geöffnet werden konnte.

\section{Ergebnisse}

\subsection{Ergebnisse der Wissensevaluierung}

\subsubsection{Regionales Wissen zur Traisen}

Die Traisen im Bereich St. Pölten weist durch Begradigung und Wasserausleitung einen „unbefriedigenden ökologischen Zustand" (Klasse 4; BMLFUW 2015) auf und wird im 2. Nationalen Gewässerbewirtschaftungsplan als „prioritärer Sanierungsraum“ definiert. Kein Jugendlicher stufte die Traisen mit einem „sehr guten“ ökologischen Zustand ein. 20 SchülerInnen (39\%) teilten der Traisen im Prä-Test einen guten ökologischen Zustand $\mathrm{zu}$, der keinen Verbesserungsbedarf zur Folge hätte. Beinahe gleich viele SchülerInnen ( $n=21 ; 42 \%)$ ordneten der Traisen die Zustandsklasse 3 (mäßiger ökologischer Zustand, $n=21$ ) zu. Nur 8 SchülerInnen (16\%) nannten im PräTest die richtige „unbefriedigende“ Einstufung des ökologischen Zustands. Ein Schüler stufte die Traisen schlechter ein (Klasse 5), ein Jugendlicher gab keine Bewertung ab (Abb. 1).

Die Auswertung der Antworten auf Frage Al) betreffend die Beeinträchtigungstypen an der Traisen zeigte einen signifikanten Unterschied zwischen beiden Klassen. Die SchülerInnen der $7 \mathrm{~N}$, die bereits im Vorgängerprojekt „FlussAu:WOW!“ beteiligt waren, nannten vor allem hydromorphologische Beeinträchtigungen (Begradigung $n=15$; Wehre, Restwasser $n=2$ ), Verschmutzung und Müll ( $n=14)$ sowie unterschiedliche intensive Landnutzungstypen $(n=13)$. Weiters wurden der „Mensch“ allgemein als übergeordneter Belastungstyp bzw. „keine Natur“ genannt. SchülerInnen der $5 \mathrm{~N}$ nannten im Mittel signifikant weniger Belastungstypen, wobei die Kategorie „Verschmutzung und Müll“ die meisten Nennungen ( $n=7)$ aufwies (Abb. 2).

Die SchülerInnen hatten zu Beginn des Projektes (Prä-Test T1) kaum ein regionales Wissen über die Traisen. Nur 7 SchülerInnen der 51 Befragten (14\%) kannten das LIFE+ Traisen-Projekt im Unterlauf. Kein einziger Jugendlicher konnte ein anderes Renaturierungsprojekt im Einzugsgebiet der Traisen im Prä-Test nennen. Im Median wurde für beide Fragen A1) „Wodurch ist der Zustand der Traisen deiner Mei- 


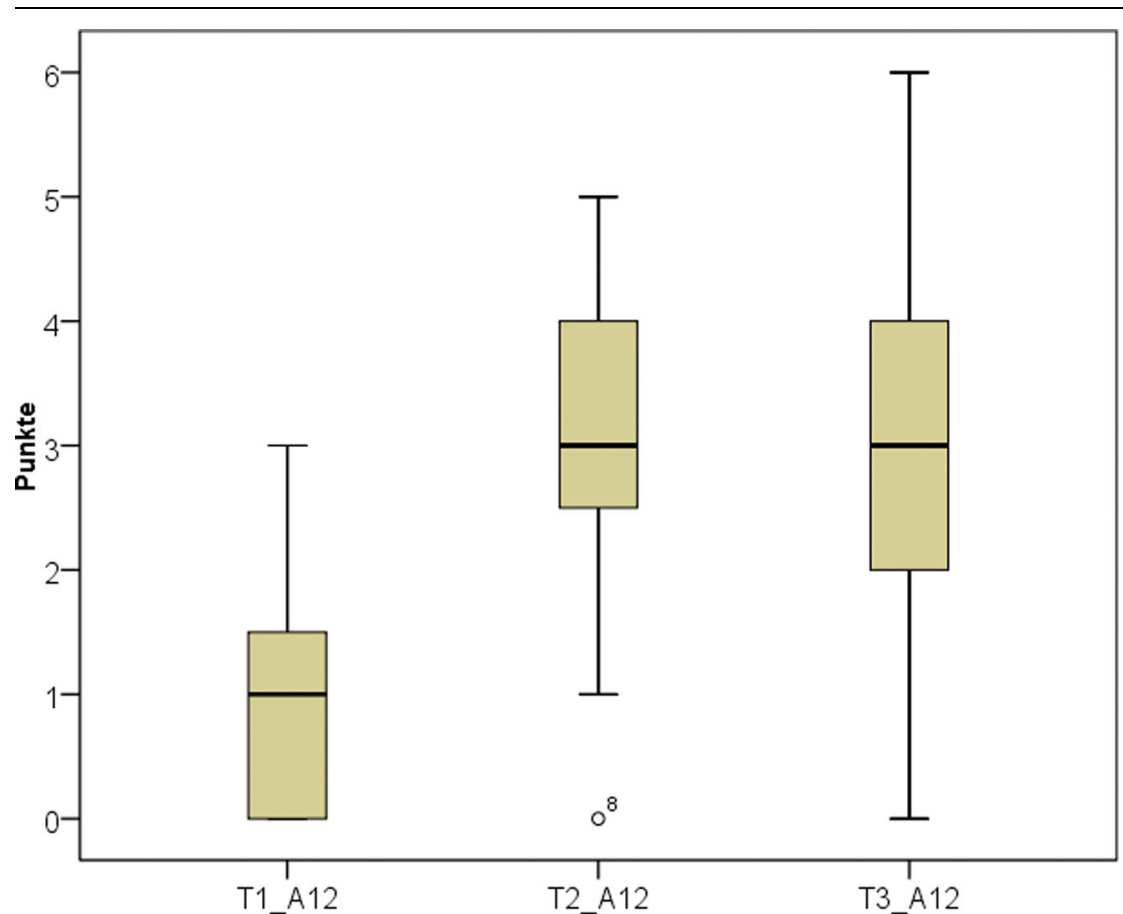

Abb. 3 Ergebnisse der Testfragen in Block A, ,regionales Wissen“ (A12) im Prä-Test (T1), Mid-Test (T2) und Post-Test (T3), N=47; max. Punkteanzahl =6; Fragen: A1) „,Wodurch ist der Zustand der Traisen deiner Meinung nach beeinträchtigt? “und A2) „Kennst du Renaturierungsprojekte im Traisen-Einzugsgebiet? Wenn ja, welche?“

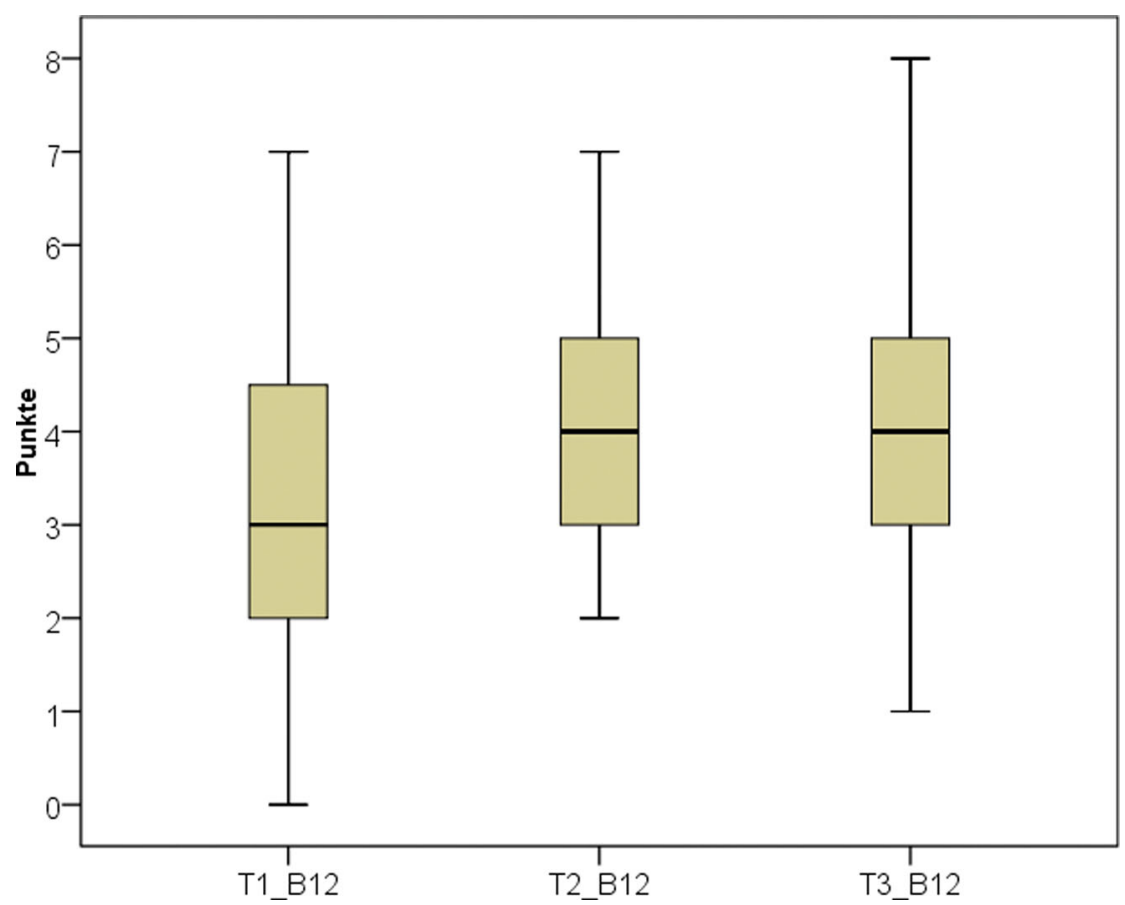

Abb. 4 Ergebnisse der Testfragen zum Block „Systemverständnis“ (B12) im PräTest (T1), Mid-Test (T2) und Post-Test (T3), N=47; max. Punkteanzahl = 8; Fragen: B1) „Warum wurden/werden Flüsse reguliert? Was verändert sich dadurch (für Menschen, Tiere und Pflanzen)?“ und B2) „Warum werden Flüsse renaturiert? Was verändert sich dadurch (für Menschen, Tiere und Pflanzen)?" nung nach beeinträchtigt?“ und A2) „Kennst du Renaturierungsprojekte im Traisen-Einzugsgebiet? Wenn ja, welche?" nur 1 Punkt von maximal 6 möglichen Punkten erreicht. Beim MidTest (T2) konnte ein signifikanter Wissenszuwachs festgestellt werden (Wilcoxon-Vorzeichen-Rang-Test, $\alpha=0.05$, $p=0.001$ ), der beim Post-Test (T3) nur mehr in Einzelfällen gesteigert wurde (Abb. 3). Hier war kein signifikanter Unterschied zwischen den Ergebnissen der 5. und der 7. Klasse feststellbar.

\subsubsection{Systemverständnis}

Die Ursache-Wirkungs-Zusammenhänge von Regulierung und Renaturierung von Fließgewässern (Block B - Systemverständnis) waren den Jugendlichen zu Beginn des Projektes weniger bekannt. Im Median erreichten sie beim Prä-Test (T1) nur 3 von 8 möglichen Punkten (vgl. Abb. 4).

Beim Mid-Test (T2) konnte ein signifikanter Wissenszuwachs festgestellt werden (Wilcoxon-Vorzeichen-RangTest, $\alpha=0.05, p=0.001)$. Beim PostTest (T3) erreichten einzelne SchülerInnen die maximale Punktezahl von 8, in der Gesamtheit konnte aber kein weiterer Wissenszuwachs dokumentiert werden (Abb. 4). Die SchülerInnen der 7. Klasse erreichten bei allen drei Tests signifikant höhere Punktezahlen als jene der 5 . Klasse.

\subsection{3 Ökosystemleistungen}

Auf die Frage C1) „Welchen Nutzen haben Flusslandschaften für den Menschen?" nannten die Jugendlichen im Prä-Test (T1) vor allem versorgende ÖSL ( $n=43$; z. B. Landwirtschaft oder Stromproduktion) und kulturelle Ökosystemleistungen $(n=42)$ wie verschiedene Freizeitaktivitäten, die am Fluss durchgeführt werden können (Abb. 5). Im Durchschnitt nannte jeder Jugendliche zwei ÖSL. Durch die Schulworkshops (Mid-Test, T2) wurde vor allem das Bewusstsein für kulturelle ÖSL $(n=87)$ bei den SchülerInnen erweitert. Nach den Feldarbeiten am Fluss und im Augebiet der Traisen wurden neuerlich vermehrt kulturelle ÖSL angeführt ( $n=97)$. Die Jugendlichen nannten weniger versorgende ÖSL $(n=30)$, währende regulierende ÖSL ( $n=14$ ), wie Klimaregulierung oder Hochwasserschutz, verstärkt angeführt wurden. Die Angabe von unterstützenden ökologischen Basisleistungen wie 


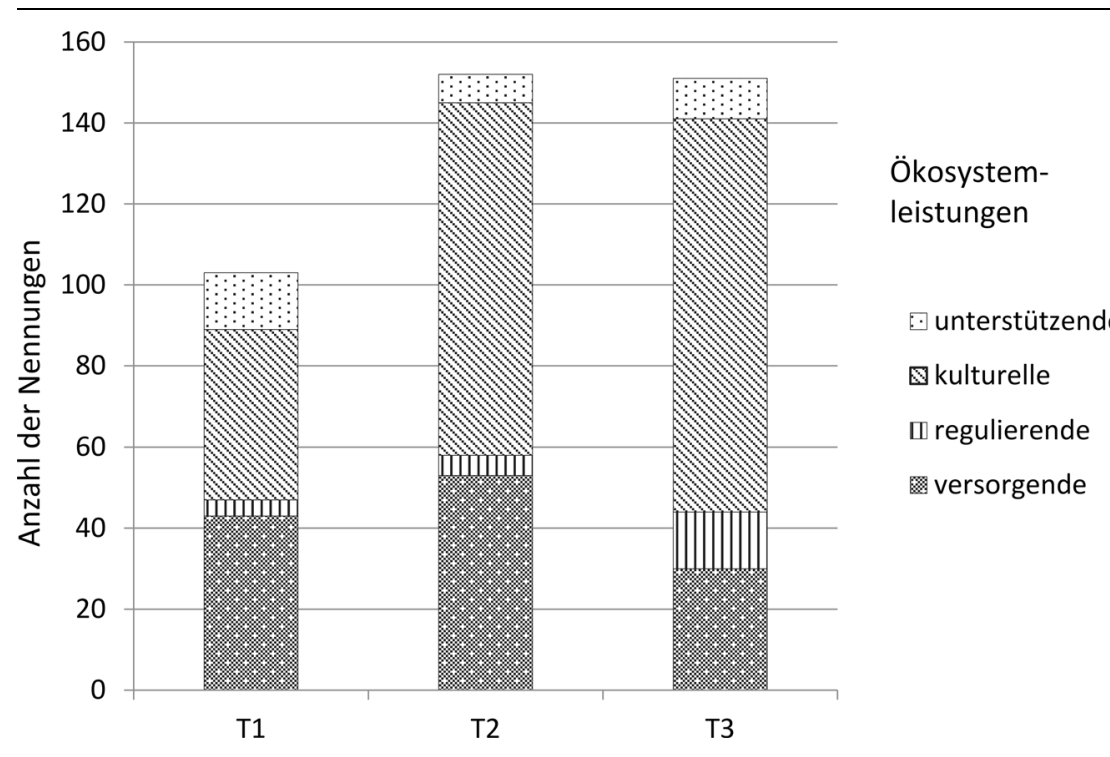

Abb. 5 Nennungen von unterschiedlichen ÖSL auf die Testfrage C1) „Welchen Nutzen haben Flusslandschaften für den Menschen?" im Prä-Test(T1), Mid-Test(T2) und PostTest (T3)

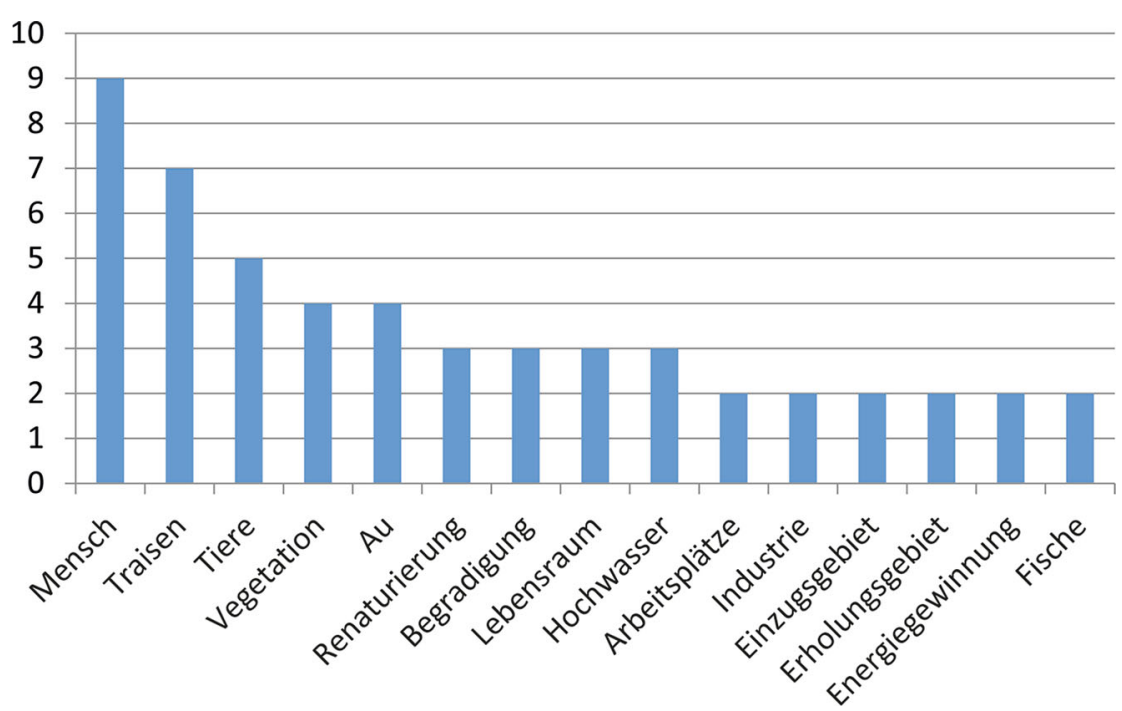

Abb. 6 Häufigkeiten der Hauptelemente in den zehn Schülerlnnen-Modellen zum Zustand der Traisen

Lebensraum, blieb bei allen drei Befragungen etwa auf gleichem Niveau. Sowohl beim Mid-Test als auch beim Post-Test nannten die SchülerInnen im Durchschnitt 3 ÖSL auf die Frage C1).

Die Auswertung aller Fragen zeigte einen signifikanten Zuwachs des Wissens und Verständnisses der SchülerInnen zwischen dem ersten und zweiten Test aufgrund der durchgeführten Schul-Workshops. Nach den Feldarbeiten konnte im Post-Test (T3) kein weiterer signifikanter Wissenszuwachs festgestellt werden. und Fische. Die Häufigkeiten der einzelnen Hauptelemente sind in Abb. 6 dargestellt. In neun der zehn Modelle wurde der Mensch als Ursache für Veränderungen in der Traisen-Flusslandschaft modelliert.

Flussbegradigungen, Brücken- und Kraftwerksbauten bzw. Bau von Fabriken und Siedlungen sowie die intensive Landwirtschaft wurden als anthropogene Eingriffe in Flusslandschaften als Haupteinflussgröße simuliert. Renaturierungen und Umweltprogramme wurden als Gegenmaßnahmen in die Modelle eingebaut. Als mögliche Motive für Handlungen, die den ökologischen Zustand bzw. die Ökosystemleistungen von Flusslandschaften potenziell negativ beeinflussen, wurden das Streben des Menschen nach Wohlstand, Lebensqualität, Erholung und sichere Arbeitsplätzen genannt. Als mögliche Auswirkungen wurden die Zunahme der Häufigkeit von Hochwässern, die Abnahme des Fischbestandes und der natürlichen Vegetation sowie Wasserverschmutzung genannt. In einigen Modellen wurden auch die durch Hochwässer verbundenen Schäden und Kosten einbezogen. Alle Modelle beinhalteten zum Großteil richtige kausale Beziehungen, wenngleich immer wieder falsche Termini verwendet wurden.

In neun Modellen wurden von den SchülerInnen-Gruppen explizit Startbedingungen gesetzt, die in der Simulation zu unterschiedlichen negativen Auswirkungen menschlicher Handlungen auf die Modellelemente einer Flusslandschaft führten. Nur in zwei Modellen wurden positive Auswirkungen auf das Ökosystem der Traisen durch die Zunahme von Umweltprogrammen bzw. durch Renaturierungen simuliert. In sieben SchülerInnen-Modellen wurden negative Auswirkungen auf die Ökosystem-Elemente, z. B. durch Bevölkerungswachstum, Zunahme von Flussbegradigungen, intensiver Landwirtschaft und steigender Umweltzerstörung, simuliert.

In Abb. 7 ist ein SchülerInnen-Modell der $7 \mathrm{~N}$ abgebildet. In Abhängigkeit von der Bevölkerungsentwicklung (Start der Simulation - grauer Pfeil) steigt u. a. der Strom- bzw. der Raumbedarf, was mit negativen Auswirkungen auf die hydromorphologischen Bedingungen in der Traisen verbunden ist, und auch deutliche Auswirkungen auf Fische und Vegetation hat. Die Entwicklungstendenzen sind in Abb. 7 durch blaue Pfeile erkennbar. Gleichzeitig steigen 


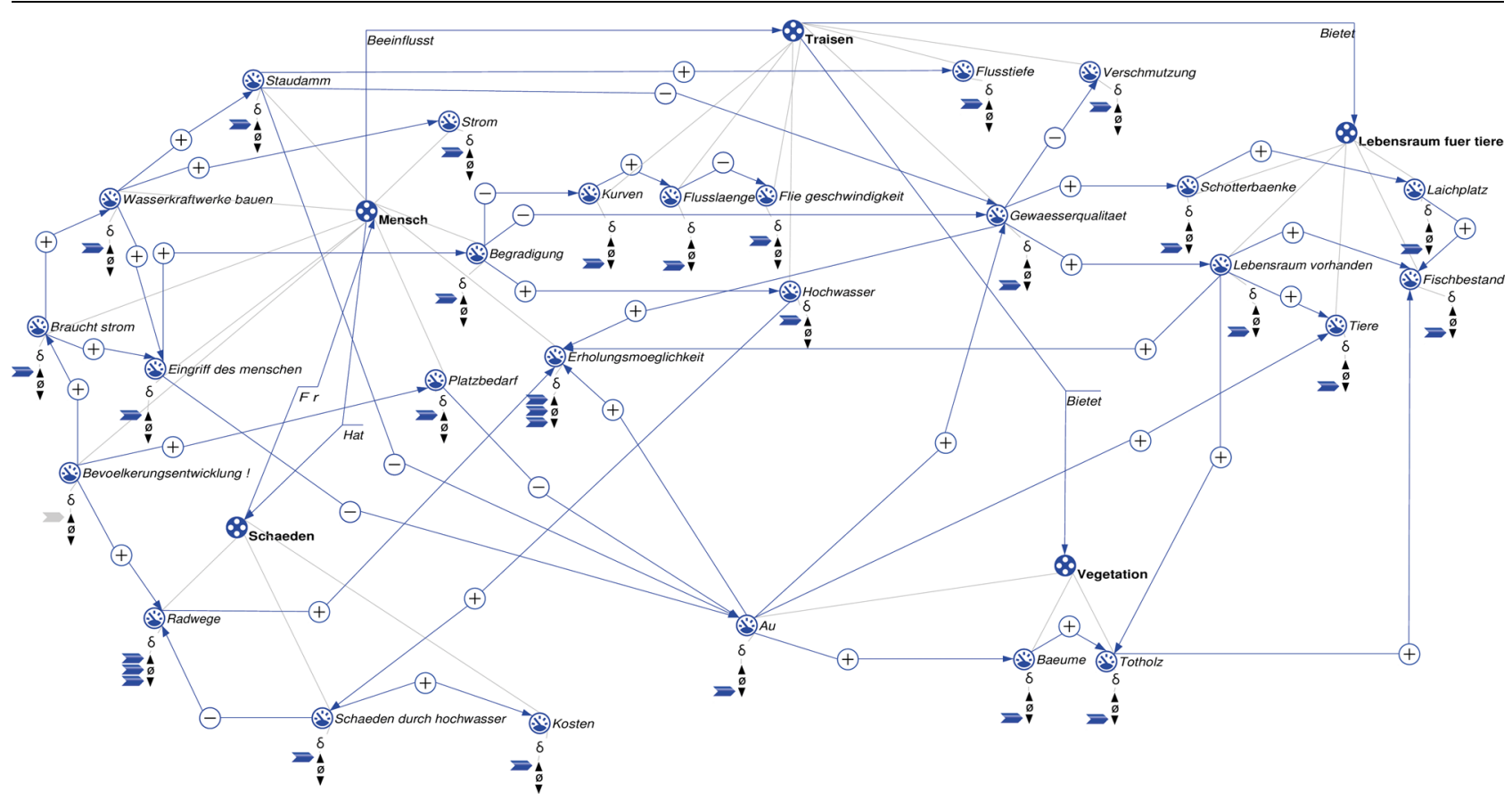

Abb. 7 Schülerlnnen-ModellzurTraisen-ModellstrukturmitSimulationsergebnissen(grauer Pfeil:StartderSimulation, blaue Pfeile zeigen Entwicklungstendenzen)

Tab. 2 Szenarien der potenziellen Entwicklung der Traisen, ausgehend von anthropogenen Beeinträchtigungen mittels unterschiedlicher Maßnahmentypen

\begin{tabular}{|l|l|l|l}
\hline Modell Nr. & Ist-Zustand & Maßnahmentyp 1 & Maßnahmentyp 2 \\
\hline 1 & Begradigung & Renaturierung & Renaturierung \\
\hline 2 & Restwasser & Entfernung der Wehranlagen & Entfernung der Wehranlagen \\
\hline 3 & Rückgang des Fischbestands & Renaturierung & Ackernutzung in Au untersagen \\
\hline 5 & Nutzungsdruck & Bauprojekte am Flussufer verhindern & Renaturierung \\
\hline 6 & Restwasser & Höhere Restwasservorschreibungen & Umweltbildung \\
\hline 8 & Schlechtes Ökosystem & Renaturierung & Renaturierung \\
\hline 9 & Verschmutzung & Baubeschränkungen & Renaturierung \\
\hline
\end{tabular}

die Hochwässer und die damit verbundenen Schäden und Kosten. Erholungsmöglichkeiten werden durch Radwege am Fluss dargestellt. In Bezug auf diese ÖSL liefert die Simulation kein eindeutiges Resultat (drei blaue Pfeile). Das kommt dadurch zustande, dass zwar durch das Vorhandensein von Radwegen die Erholungsnutzung steigt, zusätzlich wurde durch die generelle Veränderung der Flusslandschaft durch den Menschen auch eine verschlechterte Gewässerqualität angenommen, welche die Freizeitnutzung durch Radwege kontrastiert.

Die SchülerInnen-Modelle spiegelten oft die generelle Schwierigkeit wider, in Flussgebieten ein Gleichgewicht zwischen der Nutzung der Flusslandschaft durch den Menschen, den An- spruch auf intakte hydromorphologische Prozesse und die Notwendigkeit des Lebensraumes für verschiedenste Lebewesen zu finden. Viele zeigten klar das Spannungsfeld zwischen Wirtschaftswachstum, und damit intensiver Nutzung der Flusslandschaft Traisen, und den beeinträchtigten Umweltbedingungen auf.

In einer Weiterentwicklung der Modelle wurden im zweiten Modellierungs-Workshop Szenarien für das Einzugsgebiet der Traisen entwickelt. Die verschiedenen Beeinträchtigungen des Ist-Zustandes sowie die entsprechenden Maßnahmen zur Verbesserung, die von den SchülerInnen in den einzelnen Szenarien genannt wurden, sind in Tab. 2 dargestellt. In zwei Modellen wurde der „schlechte ökologische
Zustand“ bzw. das „schlechte Ökosystem" der Traisen als Ausgangssituation gewählt. Drei SchülerInnen-Modelle simulierten zwei hydromorphologische Beeinträchtigungstypen - „Restwasser“ bzw. „Begradigung“ - an der Traisen. Mit dem „Rückgang der Fischarten“ bzw. „des Fischbestandes“ arbeiteten zwei SchülerInnen-Gruppen. Zwei Modelle gingen über den Flusslauf hinaus und fokussierten auf die Beeinträchtigungstypen „Verschmutzung“ bzw. "Nutzungsdruck“ in den Aubereichen bzw. in den angrenzenden Flächen der Traisen.

Der häufigste genannte Maßnahmentyp zur Verbesserung des ökologischen und hydromorphologischen Zustandes war „Renaturierung“ ( $n=7)$, gefolgt von der „Entfernung von Wehr- 
anlagen“ ( $n=3$ ) bzw. „Einschränkung der Nutzungen“ $(n=3)$, vor allem im Ufer- und Aubereich. In einem Modell trug die Maßnahme „Umweltbildung“ indirekt zur Verbesserung der Wasserverschmutzung und des ökologischen Zustandes der Traisen bei. In den Szenarien der SchülerInnen für das gesamte Einzugsgebiet der Traisen gingen alle SchülerInnen-Gruppen vom schlechten ökologischen Ist-Zustand der Traisen aus und verbesserten den Zustand durch wirksame Maßnahmen. In sieben Szenarien wurden sogar Maßnahmenkombinationen modelliert.

Anhand der dargestellten Szenarien konnte ein deutlich zum Ausdruck gebrachtes Verständnis der wichtigsten kausalen Zusammenhänge zwischen Belastung, Zielzustand und Maßnahme bei den SchülerInnen nachgewiesen werden.

\section{Bedeutung der Ergebnisse für ein nachhaltiges}

Flusslandschaftsmanagement

\subsection{Umweltwissen und \\ Systemverständnis als Basis}

\subsubsection{Wissensvermittlung}

Wissensvermittlung von gewässerökologischen Themen ist wichtig, da bei den Jugendlichen kaum Bewusstsein über regionale Vorgänge oder Prozesse in Flusslandschaften vorhanden ist.

Die Auswertung der Wissenstests zeigte, dass regionales Wissen über den Heimatfluss und Vorgänge im Einzugsgebiet nicht im Bewusstsein der Jugendlichen verankert ist. $39 \%$ der SchülerInnen bewerteten den ökologischen Zustand der Traisen im PräTest mit "gut", wobei der Fluss gerade in St. Pölten, wo sie zur Schule gehen, stark anthropogen verändert, in einem Trapezprofil begradigt und durch Wasserausleitung beeinträchtigt ist. Hier schnitten sowohl die jüngeren als auch die älteren SchülerInnen im Mittel gleich schlecht ab. Auch europaweite Umfragen (EC 2009) zeigen, dass die Bevölkerung über Belastungen der Flusslandschaften und den Zustand der Fließgewässer kaum informiert ist.

Im Projekt „FlussAu:WOW!“ konnten wir dokumentieren, dass bei SchülerInnen „falsche“ Bilder von Belastungstypen an Flüssen vorhanden sind (Poppe et al. 2013). Dies wurde auch im gegenständlichen Projekt bestätigt, da Gewässergüte und Verschmutzun- gen als Hauptbelastungen im Traisen Flusssystem gesehen wurden. Viele SchülerInnen nannten die sichtbare Verschmutzung des Flusses und der Au mit Müll als Hauptbelastungstyp, während sie reduzierte Wasserführung, Regulierung oder Staubereiche nicht als anthropogene Eingriffe wahrnahmen. $\mathrm{Zu}$ Beginn des gegenständlichen Projekts konnten wir klar ein fehlendes Problembewusstsein der Jugendlichen zum ökologischen Zustand der Traisen belegen. Durch die Durchführung der Schulworkshops konnte dieses maßgeblich verbessert, erweitert und geschärft werden.

Um Interesse und Begeisterung der Jugendlichen hervorzurufen, muss das Thema interessieren und für die jeweilige Person, die an einem Lernprozess beteiligt ist, von Bedeutung sein (Hüther und Hauser 2012). Mit Themen wie z. B. Umweltqualität, die das eigene regionale Umfeld der SchülerInnen betreffen, bzw. mittels eigens formulierter Fragestellungen, können Jugendliche wesentlich leichter motiviert werden, aktiv und gestaltend am Unterricht teilzunehmen (Hödl 2015).

\subsubsection{Systemverständnis}

Systemverständnis ist wichtig um Ursache-Wirkungs-Zusammenhänge $\mathrm{zu}$ erkennen und nachhaltige Entscheidungen im Flussgebietsmanagement zu treffen.

Hinsichtlich des Erlernens, aber auch Verknüpfens wissenschaftlicher Konzepte und Zusammenhänge wird die Verwendung von technischen Hilfsmitteln, wie Computer-Software, zur Erstellung von repräsentativen Modellen als sehr sinnvoll und motivierend betrachtet (Jonassen und Strobl 2006). Insbesondere in der Wissenschaftserziehung hat der Prozess des Modellbildens und -testens eine große Bedeutung (Schwarz und White 2005). Qualitative kausale Modellierung wird hierbei immer öfter als wertvolles Werkzeug zur Wissenschaftserziehung betrachtet, da Lernen grundsätzlich viel mit der Entwicklung von konzeptionellem Verständnis zu tun hat (Bredeweg und Forbus 2003). Dieses bildet die Basis für das Verständnis der zugrunde liegenden Systemstrukturen und die spätere quantitative Analyse (Bredeweg und Forbus 2003; Jonassen 2003). „Qualitative konzeptuelle Modellierung auf der Basis von feldbasierter ökologischer Forschung kann Lernenden effektiv helfen, Aspekte komplexer Ökosystemfunktionen besser zu verstehen. So werden sonst abstrakte Konzepte über Interaktionen, Feedback, Subsysteme, Einflüsse und Auswirkungen zugänglicher gemacht" (Dresner und Elsner 2009).

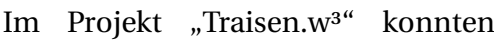
SchülerInnen damit Zusammenhänge herstellen und ihr Bewusstsein für Flusslandschaften stärken - ein Zugang der auch im nachhaltigen Flussgebietsmanagement immer mehr Anwendung findet (Hare 2011; Halbe et al. 2013). Sowohl die SchülerInnen der 5. Klasse, die verstärkt mit dem ÖSL-Konzept arbeiteten, als auch jene der 7. Klasse, die mit der Lern-Software „DynaLearn“ modellierten, zeigten nach den SchulWorkshops einen signifikanten Verständniszuwachs. Die SchülerInnen der 7. Klasse zeigten bereits zu Beginn des Projektes im Prä-Test bessere Ergebnisse und einzelne SchülerInnen dieser Klasse konnten nach allen Schulaktivitäten die maximale Punktezahl erreichen. Das ist aufgrund der bereits erfolgten Involvierung dieser SchülerInnen im Projekt „FlussAu:WOW!“, ihrer aufgrund des Alters größeren Erfahrung und ev. durch die Fokussierung auf die Modellierungsarbeit $\mathrm{zu}$ begründen. Das Modellieren, das mit der Externalisierung des eigenen persönlichen Wissensstandes beginnt und neues Wissen in konstruktiver Art und Weise in diese Modellvorstellung inkorporiert, stellt eine Aktivität dar, mit der gezielt die Komplexität und Wissenschaftlichkeit der Vorstellungen der Lernenden erweitert werden können (Erweiterung der „conceptual models“ oder „mental models“; Greca und Moreira 2000; White und Frederikson 1990; Doyle und Ford 1998). Dadurch werden die SchülerInnen letztendlich im Umgang mit komplexen und umfangreichen Informationen unterstützt und die Entwicklung einer wissenschaftlichen Denkweise über Umweltphänomene wird gefördert (Jonassen und Strobel 2006).

Die „Dynalearn“ Modelle der SchülerInnen zeigen die schwierigen komplexen Fragestellungen im Flussgebietsmanagement auf. In den zehn Szenarien zur Traisen wurde von den Jugendlichen eine Verbesserung der Umweltbedingungen als auch eine Nutzung der Flusslandschaft für Freizeit und Erholung modelliert. Steigender Strombedarf und Nutzungsdruck in Flusslandschaften wurde zwar als 
konkurrierende Einflussgrößen festgelegt, Renaturierungen, Entfernung von Wehranlagen und effektivere Gesetzgebung zum Flächenschutz in Augebieten wurden hingegen als Startimpuls für Szenarien gesetzt.

Grundsätzlich bietet das gemeinsame Erstellen analoger oder digitaler „Concept maps“ im Rahmen eines gemeinsamen „multimodalen Modellierens" einen motivierenden Einstieg in neue Themenbereiche und unterstützt dabei das gemeinsame Entwickeln und Texten (Liu et al. 2013), wie dies im Rahmen des Modellierungsworkshops anhand der am Ende der Übung verfassten verbalen Szenarien deutlich wurde. Damit konnte auch die Entwicklung der Kompetenzen in Hinsicht auf die "Scientific literacy“ (naturwissenschaftliche Grundbildung nach Bybee 1997) deutlich gefördert werden.

Gleichzeitig wurden das Verständnis über kausale Zusammenhänge sowie das grundsätzliche Systemverständnis signifikant gefördert. Die spielerische Entwicklung von Szenarien unter Einbeziehung der menschlichen Bedürfnisse und Handlungen führte $\mathrm{zu}$ einem besseren Verständnis über mögliche Handlungsalternativen in einem gegebenen sozialen Umfeld. Alle am Ende des Workshops erstellten ModellSzenarien der SchülerInnen spiegelten nachhaltige Entwicklungen der Flusslandschaft wider.

Wichtig für die Wissensvermittlung und das Lernen erscheinen multimodale Unterrichtsformen (Zitek et al. 2013; Poppe et al. 2013), die unterschiedlichste Aktivitäten (u. a. Verwendung analoger und digitaler Systemzugänge, z. B. mit der „DynaLearn“-Lern-Software, Diskussionen, Vorträge, Feedback-Runden, Schreiben von Blog-Beiträgen, Befragungen, Feldarbeiten, selbstständig durchgeführte Gruppenarbeiten, Erstellung eines Kurzfilms) in der Zusammenarbeit mit den SchülerInnen integrieren. Interessanterweise konnte bei der Punkteauswertung der Wissenstests kein signifikant erweitertes Systemverständnis durch die Feldarbeiten der SchülerInnen dokumentiert werden. Bei der Analyse der einzelnen Antworten wurden aber nach den Feldarbeiten neue Begriffe verwendet. Beim Themenblock der ÖSL wurden durch die Arbeit im Augebiet der Traisen neue Funktionen von Flusslandschaften wie Hochwasserretention oder Klimaregulation von den SchülerInnen wahrgenommen und in den Testantworten an- geführt, während andere, ursprünglich genannte ÖSL weggelassen wurden.

Gemeinsam mit den SchülerInnen des BG/BRG St. Pölten wurde während der Workshops und der Feldarbeiten ein Kurzfilm erstellt ${ }^{5}$. Hier ist der Spaß an der Feldarbeit deutlich ersichtlich, was auch im Feedback der SchülerInnen an das Projektteam gemeinsam mit den vielfältigen, abwechslungsreichen Schulaktivitäten klar als positive Aspekte des Projektes angesprochen wurde.

\subsubsection{Social-learning-Aktivitäten}

Social learning ist ein wichtiger Aspekt im nachhaltigen Flussgebietsmanagement (Pahl-Wostl et al. 2008; Koontz 2014) und wird durch interaktives, kollaboratives Modellieren und Gruppenaktivitäten gefördert (Hare 2011; Halbe et al. 2013). Zusätzlich zu dem Ziel des gemeinsamen Arbeitens war es wichtig, den SchülerInnen spannende Möglichkeiten des gemeinsamen Lernens zu bieten, bei denen gleichzeitig der Wissensaustausch, aber auch soziale Kompetenzen wie Kommunikationsfähigkeit, Kooperation, Konflikt- und Teamfähigkeit gefördert werden sollten. Dies wurde von einzelnen SchülerInnen im direkten Feedback an das Forschungsteam als bereichernde Art des Lernens genannt. Die durch die SchulWorkshops erworbenen Kompetenzen können z. B. bei zukünftigen Partizipationsprozessen eingesetzt werden.

\subsection{Das Konzept der ÖSL als geeignetes Kommunikationsinstrument}

Die Ergebnisse dokumentieren, dass das Konzept der ÖSL eine geeignete Methode zur Wissensvermittlung von komplexen Zusammenhängen in Flusslandschaften darstellt. Damit bestätigen sich die Ergebnisse einer Untersuchung an den Flusslandschaften Enns und Drau (Böck et al. 2015), in der InterviewpartnerInnen aus unterschiedlichen Fachbereichen das ÖSLKonzept als vielversprechendes Instrument für Kommunikationszwecke und Umweltbildung einstuften. Es wurde angenommen, dass das Konzept das Verständnis für die Zusammenhänge zwischen Gesellschaft und Natur verbessern und Neugier und Interesse fördern kann (Böck et al. 2015).

${ }^{5}$ http://www.traisen.net/?page_id=452.
$\mathrm{Zu}$ Beginn des Projekts waren vor allem kulturelle und versorgende ÖSL im Bewusstsein der Jugendlichen und wurden auch im Prä-Test genannt. Die Flusslandschaft wurde als Ort für Freizeitaktivitäten (Freunde treffen, spazieren gehen, fischen) gesehen, aber auch als bedeutsam für die Landwirtschaft oder zur Stromproduktion wahrgenommen. Interessanterweise wurde von den SchülerInnen nach den Feldarbeiten eine Vielzahl von kulturellen ÖSL genannt sowie neue regulierende Leistungen. Diese wurden verstärkt durch die Feldarbeit an der Traisen und im Augebiet des LIFE+ Projekts wahrgenommen. Die Bedeutung kultureller Leistungen zeigt sich auch in früheren Untersuchungen zur Wahrnehmung von ÖSL durch unterschiedliche Stakeholder-Gruppen (Chiari 2010; Böck et al. 2013; Hauck et al. 2013). Diese steht jedoch im starken Widerspruch zur Berücksichtigung dieser Leistungen in der Praxis (Daniel et al. 2012; Schaich et al. 2010). Hier wird den Versorgungsleistungen, denen in dieser Studie von den Jugendlichen ebenfalls eine hohe Bedeutung zugemessen wurde, der Vorrang gegeben. Es sollte jedoch nicht vergessen werden, dass besonders kulturelle Leistungen eine entscheidende Rolle beim Management der Interaktionen zwischen Mensch und Umwelt sowie bei der Erreichung öffentlicher Unterstützung für den Schutz von Ökosystemen einnehmen (Daniel et al. 2012).

\subsection{Ansätze zur Partizipation: Einbeziehung der Jugend wichtig}

Europaweite Umfragen (EC 2012) zeigten, dass gerade die lokale Bevölkerung wenig über die Möglichkeit der Partizipation bei Planungen im Flussgebietsmanagement weiß. Neun von zehn Personen ist der Begriff eines Gewässerbewirtschaftungsplans nicht bekannt. In Österreich gaben $93 \%$ aller Befragten $(n=1003)$ an, dass sie sich nicht an der Entwicklung der Gewässerbewirtschaftungspläne im Rahmen von Konsultationsprozessen beteiligt haben, wobei davon $45 \%$ bei entsprechender Information teilgenommen hätten (EC 2012).

Einige Studien (CIS 2003; de Stefano 2010; Demetropoulou et al. 2010) zeigten, dass Partizipationsprozesse auf lokaler und regionaler Ebene besonders geeignet sind, um das Umweltwissen, Systemverständnis und das Interesse 
der Bevölkerung zu erhöhen. Gerade bei jungen Menschen ist es wichtig, das Interesse für Umwelthemen zu fördern und eine mögliche Beteiligung an Entscheidungen $\mathrm{zu}$ forcieren (Nguyen und Bosch 2014). Je mehr Jugendliche frühzeitig in einen Partizipationsprozess eingebunden werden, der ihnen Handlungsoptionen zur Verbesserung der Umweltsituation ermöglicht, desto größer sind die Chancen auf ein bewusstes Umwelthandeln in späteren Jahren (Kromer und Zuber 2003). „Stimme der Jugend ${ }^{6 “, ~ „ s t r u k t u r i e r-~}$ ter Dialog7“, „Jugend in Aktion ${ }^{8 “}$ oder „Youth for the Alps“ (CIPRA 2013) sind österreichische bzw. alpen- oder europaweite Initiativen, die junge Menschen aktiv in Entscheidungsprozesse im politischen und sozialen Umfeld einbinden. Diese Initiativen sollten gezielt

${ }^{6}$ https://stimme-der-jugend.at.

7 http://www.strukturierter-dialog.at.

${ }^{8}$ http://www.jugendinaktion.at. auch auf ökologische Themenfelder ausgeweitet werden, damit junge Menschen zukünftig als BewohnerInnen und NutzerInnen der Flusslandschaften nachhaltige Planungen und Maßnahmen unterstützen, sich aktiv an der Entscheidungsfindung beteiligen, und damit Verantwortung für die Gestaltung ihrer Umwelt übernehmen können.

\section{Danksagung}

Dieser Artikel wurde im Rahmen des „Sparkling Science“-Forschungsprojekts „Traisen.w ${ }^{3}$ “ verfasst. Besonderen Dank möchten wir allen Schülerinnen und Schülern der $5 / 6 \mathrm{~N}$ und $7 / 8 \mathrm{~N}$ des BG/BRG St. Pölten und dem PädagogInnen-Team aussprechen. Die WissenschaftlerInnen wurden von Mag. Andrea Heidenreich, Mag. Martin Schrittwieser und Mag. Roman Kurz-Aigner im Schulbetrieb tatkräftig unterstützt. Vielen Dank auch an Dr. Helmut Wimmer (Verbund AG), Mag. Florian Seidl (Verbund AG), Dr. Thomas Kaufmann (Büro Freiwasser), DI Reinhard Barbl
(Büro Steinwender \& Partner GmbH) sowie Ing. Günther Lindmayr (Forstamt Stift Herzogenburg), die uns bei den Kartierungsarbeiten an der Traisen unterstützten bzw. diese ermöglichten.

Open access funding provided by University of Natural Resources and Life Sciences Vienna (BOKU)

Open access funding provided by University of Natural Resources and Life Sciences Vienna (BOKU).

Open Access Dieser Artikel wird unter der Creative Commons Namensnennung 4.0 International Lizenz (http:// creativecommons.org/licenses/by/4. $0 /$ deed.de) veröffentlicht, welche die Nutzung, Vervielfältigung, Bearbeitung, Verbreitung und Wiedergabe in jeglichem Medium und Format erlaubt, sofern Sie den/die ursprünglichen $\mathrm{Au}$ tor(en) und die Quelle ordnungsgemäß nennen, einen Link zur Creative Commons Lizenz beifügen und angeben, ob Änderungen vorgenommen wurden.

\section{Literatur}

Bilharz, M., Gräsel, C. (2006): Gewusst wie Strategisches Umwelthandeln als Ansatz zur Förderung ökologischer Kompetenz in Schule und Weiterbildung [ Einzelbeitrag ]. Bildungforschung, 3, 1-32.

Böck, K., Muhar, A, Oberdiek, J., Muhar, S. (2013): Die Wahrnehmung von fließgewässerbezogenen „Ökosystemleistungen“ und Konfliktpotenzialen am Fallbeispiel "Flusslandschaf Enns“. Österreichische Wasser- und Abfallwirtschaft 11-12.

Böck, K., Muhar, A., Muhar, S., Polt, R. (2015) The Ecosystem Services Concept: Gaps between Science and Practice in River Landscape Management. GAIA 24(1): 32-40

Bredeweg, B., Forbus, K. D. (2003): Qualitative Modeling in Education. AI Magazine, 24, 35-46. Bredeweg, B., Liem, J., Beek, W., Linnebank, F, Gracia, J., Lozano, E., Wißner, M., Bühling, R., Salles, P., Noble, R., Zitek, A., Borisova, P., Mioduser, D. (2013): DynaLearn - An intelligent learning environment for learning conceptual knowledge. AI Magazine 34(4):46-65.

Bückmann, W. (2015): Die Vision der UNO für die Zukunft der Welt: die 2030 Agenda für eine nachhaltige Entwicklung. FAGUS Schriften 17 $115 S$.

Bundesministerium für Land- und Forstwirtschaft, Umwelt und Wasserwirtschaft (BMLFUW) (Hrsg., 2015): Nationaler Gewässerbewirtschaftungsplan (NGP) 2015. Entwurf, Stand Jänner 2015.

Bybee, R. (1997): Towards an Understanding of Scientific Literacy. In: Gräber, W. \& Bolte, C. (Hrsg.), Scientific Literacy, Kiel, 37-68.

Chan, K. M. A., Satterfield, T., Goldstein, T. Goldstein, J. (2012): Rethinking ecosystem services to better address and navigate cultural values. Ecological Economics 74: 8-18.

Chiari, S. (2010): Raumbedarf für multifunktionale Flusslandschaften-potentielle Synergien zwischen ökologischen Erfordernissen und den
Bedürfnissen der Freizeit- und Erholungsnutzung. Doktoratskolleg Nachhaltige Entwicklung (dokNE), Universität für Bodenkultur, Wien. Dissertation: 228.

Chuchmáková, L. (2009): Assessment of pupils' perception of ecosystem services as a basis for designing environmental education strategies in the Baviaanskloof valley in South Africa Wageningen University, Wageningen. Master Thesis: 103.

Commission Internationale pour la Protection des Alpes (CIPRA, ed. 2013): Youth Participation in the Alps. Schaan. 109S.

Common Implementation Strategy (CIS 2003) Common Implementation Strategy for the Water Framework Directive (2000/60/EC): Public Participation in relation to the Water Framework Directive.

Daniel, T. C., Muhar, A., Arnberger, A, Aznar, O. Boyd, J. W., Chan, K. M., Costanza, R., Elmqvist, T., Flint, C. G., Gobster, P. H., Gret-Regamey, A., Lave, R., Muhar, S., Penker, M., Ribe, R. G., Schauppenlehner, T., Sikor, T., Soloviy, I. Spierenburg, M., Taczanowska Tam, J., von der Dunk, A. (2012): Contributions of cultural services to the ecosystem service agenda. PNAS

Demetropoulou, L., Nikolaidis, N., Papadoulakis, V., Tsakiris, K., Koussouris, T., Kalogerakis, N., Koukaras, K., Chatzinikolaou, A., Theodoropoulos, K. (2010): Water Framework Directive Implementation in Greece: Introducing Participation in Water Governance - the Case of the Evrotas River Basin Management Plan. Environmental Policy and Governance, 20, 336-349. http://doi.org/10.1002/eet.553

De Stefano, L. (2010): Facing the water framework directive challenges: a baseline of stakeholder participation in the European Union. Journal of Environmental Management. http://doi.org/ 10.1016/j.jenvman.2010.02.014
Doyle, J. K., Ford, D. N. (1998): Mental models concepts for system dynamics research. System dynamics review 14.1: 3-29.

Dresner, M., Elser, M. (2009): Enhancing science teachers' understanding of ecosystem interactions with qualitative conceptual models. Teaching Issues and Experiments in Ecology, Vol. 6: Research (online source)

Eder, R., Arnberger, A. (2010): Urban pupils views on floodplains - results from a photo-diary. In: Findeis G, Arnberger A, Eder R, Schuster K (eds.). Urban People Meet Urban Forests. Conference Proceedings of the 13th European Forum on Urban Forestry, pp. 23

European Commission (EC, 2009): Flash Eurobarometer on Water. Survey 26.-31.1.2009. Summary of Austrian Results. Flash EB Series 261. Gallup Organisation.

European Commission (EC, 2012): Attitudes of Europeans towards water-related issues. Flash Eurobarometer 344. 153S.

Frischknecht-Tobler, U., Nagel, U., Seybold, H. (2008): Systemdenken. Wie Kinder und Jugendliche komplexe Systeme verstehen lernen: 164S. Greca, I. M., Moreira, M. A. (2000): Mental models, conceptual models, and modelling. International Journal of Science Education 22.1: 1-11. Halbe, J., Pahl-Wostl, C., Sendzimir, J., Adamowski, J. (2013): Towards adaptive and integrated management paradigms to meet the challenges of water governance. Water Science and Technology, 67.11, 2251-2260. http://doi. org/10.2166/wst.2013.146

Hare, M. (2011): Forms of Participatory Modelling and its Potential for Widespread Adoption in the Water Sector. Environmental Policy and Governance, 21, 386-402. http://doi.org/10.1002/ eet 590

Hauck, J., Görg, C., Varjopuro, R., Ratamäki, O., Jax, K. (2013): Benefits and limitations of the ecosystem services concept in environmental policy and decision making: Some stakeholder 
perspectives. Environmental Science and Policy 25: 13-21.

Hödl, R. (2015): Lernen durch Begeisterung Diplomarbeit. Universität Wien, Institut für Geographie.140S.

Hüther, G., Hauser, U. (2012): Jedes Kind ist hoch begabt. Die angeborenen Talente unsere Kinder und was wir aus ihnen machen. München.

Jonassen, D. (2003): Using cognitive tools to represent problems. Journal of Research on Technology in Education 35(3):362-381.

Jonassen, D., Strobel, J. (2006): Modeling for Meaningful Learning. In: D. Hung, M.S. Khine (Hrsg.): Engaged Learning with Emerging Technologies. Dordrecht: Springer Netherlands.

Koontz, T. M. (2014): Social learning in collaborative watershed planning: the importance of process control and efficacy. Environmenta Planning and Management, 57(10), 1572-1593. http://doi.org/10.1080/09640568.2013.820658 Kromer, I., Zuba, R. (2003): Jugend im Wandel. Umwelt Und Bildung, 23-25.

Liu, W.-Y. I., Chao, Y.-C. J., Wu, W.-C. V. (2013) Using Concept Maps to Enhance EFL Students' Collaborative Writing: Paper-based and computer-mediated approaches. In: L.-H.e.a. Wong (ed.): 21st International Conference on Computers in Education. Indonesia Asia-Pacific Society for Computers in Education.

MEA (2005): Ecosystems and Human Wellbeing: A Framework for Assessment. Millenium Ecosystem Assessment Washington, D.C. World Resources Institute: 266S.

Millward, A., Mostyn, B. (1989): People and nature in cities. The social aspects of planning and managing natural parks in urban areas. Joint $\mathrm{Na}$ ture Conservation Committee, Petersborough, United Kingdom.

Nguyen, N. C., Bosch, O. J. H. (2014): The Art of Interconnected Thinking: Starting with the Young, Challenges, 5, 239-259. http://doi.org/ $10.3390 /$ challe5020239
Organisation for Economic Co-operation and Development (OECD, 2009): Green at Fifteen? How 15-Year-Olds perform in environmenta science and geoscience in PISA 2006. http:// doi.org/10.1787/9789264063600-en

Pahl-Wostl, C., Tàbara, D., Bouwen, R., Craps, M., Dewulf, A., Mostert, E. et al (2008): The importance of social learning and culture for sustainable water management. Ecological Economics, 64, 484-495. http://doi.org/10.1016/j. ecolecon.2007.08.007

Plieninger, T., Dijks, S., Oteros-Rozas, E., Bieling, C. (2013): Assessing, mapping, and quantifying cultural ecosystem services at community level. Land Use Policy 33/0: 118-129.

Poppe, M., Zitek, A., Scheikl, S., Preis, S., Mansberger, R., Grillmayer, R., Muhar, S. (2013): Erfassen von Ursache-Wirkungs-Beziehungen in Flusslandschaften: Vermittlung von Systemwissen in Schulen als Beitrag für ein nachhaltiges Flussgebietsmanagement. Österreichische Wasser- und Abfallwirtschaft. doi:10.1007/s00506013-0119-x

Poppe, M., Zitek, A., Stelzhammer, M., Bredeweg, B., Muhar, S. (2011): How can we increase causal and structural system understanding in environmental science education? First evaluation results of a conceptual modelling software (DynaLearn) in Austria. Geographical Research Abstracts Vol. 13, EGU 2011-5725, Vienna.

Richtlinie 2007/60/EG des Europäischen Parlaments und des Rates vom 23. Oktober 2007 übe die Bewertung und das Management von Hochwasserrisiken

Richtlinie 2003/35/EG des Europäischen Parlaments und des Rates vom 26. Mai 2003 über die Beteiligung der Öffentlichkeit bei der Ausarbeitung bestimmter umweltbezogener Pläne und Programme und zur Änderung der Richtlinien 85/337/EWG und 96/61/EG des Rates in Bezug auf die Öffentlichkeitsbeteiligung und den Zugang zu Gerichten
Richtlinie 2000/60/EG des Europäischen Parlamentes und des Rates vom 23.10.2000 zur Schaffung eines Ordnungsrahmens für Maßnahmen der Gemeinschaft im Bereich des Wasserpolitik, ABI 2000 L327/ 1.

Schaich, H., Bieling, C., Plieninger, T. (2010): Linking Ecosystem Services with Cultural Landscape Research. GAIA 19/4: 269-277.

Schwarz, C. V., White, B. Y. (2005): Metamodeling Knowledge: Developing Students' Understanding of Scientific Modeling. Cognition and Instruction, 23, 165 - 205.

SWEDESD (s.a): Introduction to the SWEDESD Education Programme: Ecosystem Services, Strong Sustainability and Agency (ESSA) -Learning for change.

Tapsell, S., Tunstall, S., House, M., Whomsley, J., Macnaghten, P. (2001): Growing up with rivers? Rivers in London children's worlds. Area 33.2: 177-189.

United Nations educational, scientific and cultural Organisation (UNESCO, 2011): Education for sustainable development: an expert review of processes and learning. Monitoring and Evaluation. 132S.

United Nations (UN, 2015): Transforming our world: the 2030 Agenda for Sustainable Development (Vol. 16301). A/RES/70/1.35S

White, B. Y., Frederiksen, J. R. (1990): Causal model progressions as a foundation for intelligent learning environments. Artificial intelligence 42.1: 99-157.

Zitek, A., Poppe, M., Stelzhammer, M., Muhar, S., Bredeweg, B. (2013): Learning by Conceptual Modeling-Changes in Knowledge Structure and Content. IEEE T LEARN TECHNOL. 6(3): 217-227.

Zitek, A., Schmutz, S., Preis, S., Bredeweg, B. Salles, P., Muhar, S. (2009): Evaluating the potential of qualitative reasoning models to contribute to a sustainable catchment management. Ecological Informatics 4(5): 381-395. 